\title{
Numerical and experimental verification of initial, transitional and turbulent regions of free turbulent round jet
}

\author{
Shahriar Ghahremanian ${ }^{1}$ and Bahram Moshfegh ${ }^{2}$ \\ Division of Energy Systems, Department of Management and Engineering, Linköping University, Sweden \\ Department of Building, Energy and Environmental Engineering, Faculty of Engineering and Sustainable \\ Development, University of Gävle, Sweden
}

\begin{abstract}
Three-dimensional simulation of the whole domain (initial, transition and fully developed regions) of round jet is essential in order to predict and to study the flow behavior of multiple jets (e.g. confluent jets). According to authors knowledge, numerical prediction of round jet with RANS models that has been presented by other researchers, are only in twodimensional (axisymmetric) and mostly for the fully developed region. The inlet boundary conditions, inlet velocity profile, turbulent kinetic energy and its dissipation rate at the diffuser exit has been governed from an earlier verified numerical simulation. In the present paper, results of three-dimensional modeling of isothermal, free, turbulent round jet with two two-equation (Low Re $k-\epsilon$ and SST $k-\omega)$, a transition three-equation $(k-k l-\omega)$ and a transition four-equation (SST) eddy-viscosity turbulence models with resolved inlet profiles are compared and validated with hot-wire anemometry. This study shows that numerical simulation of round jet with SST $k-\omega$ gives good agreement with measured mean longitudinal velocities, while transition models could only predict the initial region of round jet.
\end{abstract}

\section{Introduction}

$M^{2}$ ANY engineering applications use a complex combination of round jets due to their capacity for high mixing process, fully turbulent characteristics and ease of production. For new industrial design, engineers need suitable and accurate three-dimensional CFD codes to predict the round-jet dynamics (e.g. velocity components) in the proximal regions, which contain recirculation due to entrainment and occurrence of transition to turbulence.

Round jets are also fundamentally significant as a free shear flow due not only to the self-similarity feature but also to the axisymmetric configuration (symmetry in statistics around streamwise centerline). Free round jet can be defined as fluid spread from a circular nozzle through a stationary medium. Entrainment occurs from the edge of round jet, i.e. ambient fluids enter into the jet flow, while the velocity profile becomes wider in spreading angle and lower in magnitude as distance from nozzle increases. This diverging form of mixing layers intersects at the edge of the nozzle and creates a virtual origin. Round jet has three major regions: initial (flow development) region, transitional region and fully developed turbulent mixing layer (self-similar region). In the first two regions, centerline streamwise velocity remains constant or sharply increases depending on diffuser, nozzle type and inlet velocity profile, etc $[1,2]$. The initial and transition regions of a turbulent, free round jet have been investigated by Sami et al. [3], Hill [4], Boguslawski and Popiel [5], Obot et al. [6], Quinn and Militzer [2] and Olsson and Fuchs [1].

Table 1 shows a summary of provided data and nozzle type for the above-mentioned studies. Some other experimental and numerical investigations regarding jet noise suppression were carried out by Davies et al. [7], Bradshaw et al. [8] and Ko and Davies [9] and Lau and Fischer [10]. The coherent large-scale structures of the jet have been studied by Crow and Champagne [11], Hussain [12] and Dimotakis et al. [13]. Analytical solutions of the

\footnotetext{
${ }^{1} \mathrm{PhD}$ student, Division of Energy Systems, Department of Management and Engineering, Linköping University, Sweden and Department of Building, Energy and Environmental Engineering, Faculty of Engineering and Sustainable Development, University of Gävle, Sweden, Shahriar.Ghahremanian@liu.se, shrghn@hig.se, and AIAA student Member.

${ }^{2}$ Head, Division of Energy Systems, Department of Management and Engineering, Linköping University, Sweden and Professor, Department of Building, Energy and Environmental Engineering, Faculty of Engineering and Sustainable Development, University of Gävle, Sweden, Bahram.Moshfegh@liu.se, bmh@ @ig.se.
}

American Institute of Aeronautics and Astronautics 
governing partial differential equations are not available for the initial and transition regions. In the self-similar region, inverse decay of centerline mean velocity lies on a straight line that can yields the virtual origin and spreading rate of round jet. Decay of centerline velocity for exit Reynolds number is

$$
\frac{U}{U_{\max }}=\text { Constant }\left(\frac{d_{0}}{y-y_{p}}\right)
$$

where $y=y_{p}$ is the virtual origin of the jet flow and A is centerline velocity decay constant. A comprehensive study of previous works on the fully developed region of round jet is presented by Pope [14]. Some contributions to the understanding of the fully developed region of round jet have been experimentally made by Wygnanski and Fiedler [15], Rodi [16], Panchapakesan and Lumley [17] and Hussein et al. [18]. Analytical solution of the jet is possible in the fully developed region due to self-similarity exhibition of mean flow and turbulence structure. Solution of closed form of the governing partial differential equations has been done, for example, by Rajaratnam [19] and Schlichting [20]. Numerical studies using the finite difference method of the fully developed region have been done, for example, by Spalding [21], Launder et al [22], Pope [23], Givi and Ramos [24], Cho and Chung [25] and Ghirelli [26]. Characteristic decay region, which is available in plane jets, is so short that can be neglected.

Table 1. Provided data and nozzles type in other studies

\begin{tabular}{ccc}
\hline \hline Reference & Provided information & Nozzle type \\
\hline Sami et al [3] & Mean flow, mean static pressure, turbulence data & $\begin{array}{c}\text { Smoothly contoured nozzle } \\
\text { Short converging round nozzle } \\
\text { Hill [4] }\end{array}$ \\
$\begin{array}{c}\text { Moguslawski entrainment } \\
\text { Popiel [5] }\end{array}$ & Mean streamwise velocity, turbulence data & $\begin{array}{c}\text { Fully developed turbulent flow } \\
\text { from a long circular pipe }\end{array}$ \\
Obot et al. [6] & Mean streamwise velocity, spreading rate, mass & Square-edged round slot \\
Quinn and Militzer & entrainment rate & Sharp-edged round slot, smoothly \\
[2] & turbulent data, mean static pressure & contracting round nozzle \\
\hline \hline
\end{tabular}

When the flow leaves the leading edge of the nozzle, it becomes unstable due to disturbances of velocity profiles. Therefore providing accurate inlet boundary conditions is important for the correct prediction of the initial and transitional regions. Two length scales can characterize considerable-complicated round jet: Inlet boundary shear-layer thickness (e.g. momentum thickness) and nozzle diameter. Near field large-vortices scale depends on inlet momentum thickness while jet diameter is the controlling length scale afterward. While inlet boundary conditions of round jet affect initial large-scale vortices by forming axisymmetric vortex rings close to nozzle exit and helical instabilities at downstream (sometimes in a transition between two states [13]), length of becoming selfsimilar can be different for mean flow properties from higher order moments. Zaman and Hussain [27] looked at large scales of round jets and reported that structures contour at a certain distance from leading edge of nozzle are not noticeably dependent on a certain range of Reynolds number of inlet $\left(R e_{d_{0}}\right)$. Reviews of previous investigations of turbulent round free jet assess effects of inlet boundary conditions. Gouldin et al. [28] completely summarized the round jet data from previous experimental results and attributed the cause of variation in spreading rate, virtual origin and centerline mean velocity to inlet boundary conditions. Then two strategies are suggested regarding the problem of choosing inlet boundary conditions: design a well-defined laminar inlet condition or turbulent inlet boundary layer. Transition modeling of large scales from laminar to turbulence is a difficulty for the first idea, while the requirement of extensive measurements (e.g. means, higher moments and their correlations) for specifying the turbulent boundary is a difficulty for the second alternative. Despite the below-mentioned problem of modeling of initial, transitional and fully developed regions, they presented perfectly matched two-dimensional numerical prediction results with experiments of a free shear flow issued from a turbulent pipe. The twentieth century belief that "turbulence forgets its origins" or universal self-similarity has been analytically reviewed and objected to by George [29], George and Davidson [30] and Boersma et al. [31]. They [30] related production of asymptotic dependence of inlet boundary conditions to necessary physics (vorticity production, convection and diffusion) by giving some examples. They [30] also showed that mean velocity profile and Reynolds stress profiles are independent of all inlet conditions in self-preserving region of wakes and all similar canonical flows but not growth rate and other moment profiles. Experiments on circular jets done by Antonia and Zhao [32] show a proof for the mentioned independency. The study by George and Davidson [30] stated that single-point Reynolds averaged Navier-Stokes (RANS) turbulence models are not able to perform the asymptotic effects provided by the inlet boundary conditions. While in contrast, Large Eddy Simulation (LES) overcome this problem by resolving the large turbulence scales which are important for canonical flows. 
In the present paper, inlet velocity profile, turbulent kinetic energy and its dissipation rate (see Figure 1) of round jet are taken from a verified numerical simulation [33], and a good agreement between measurement and the results of whole field simulation of free round jet by RANS turbulence models with provided inlet boundary condition can be observed.

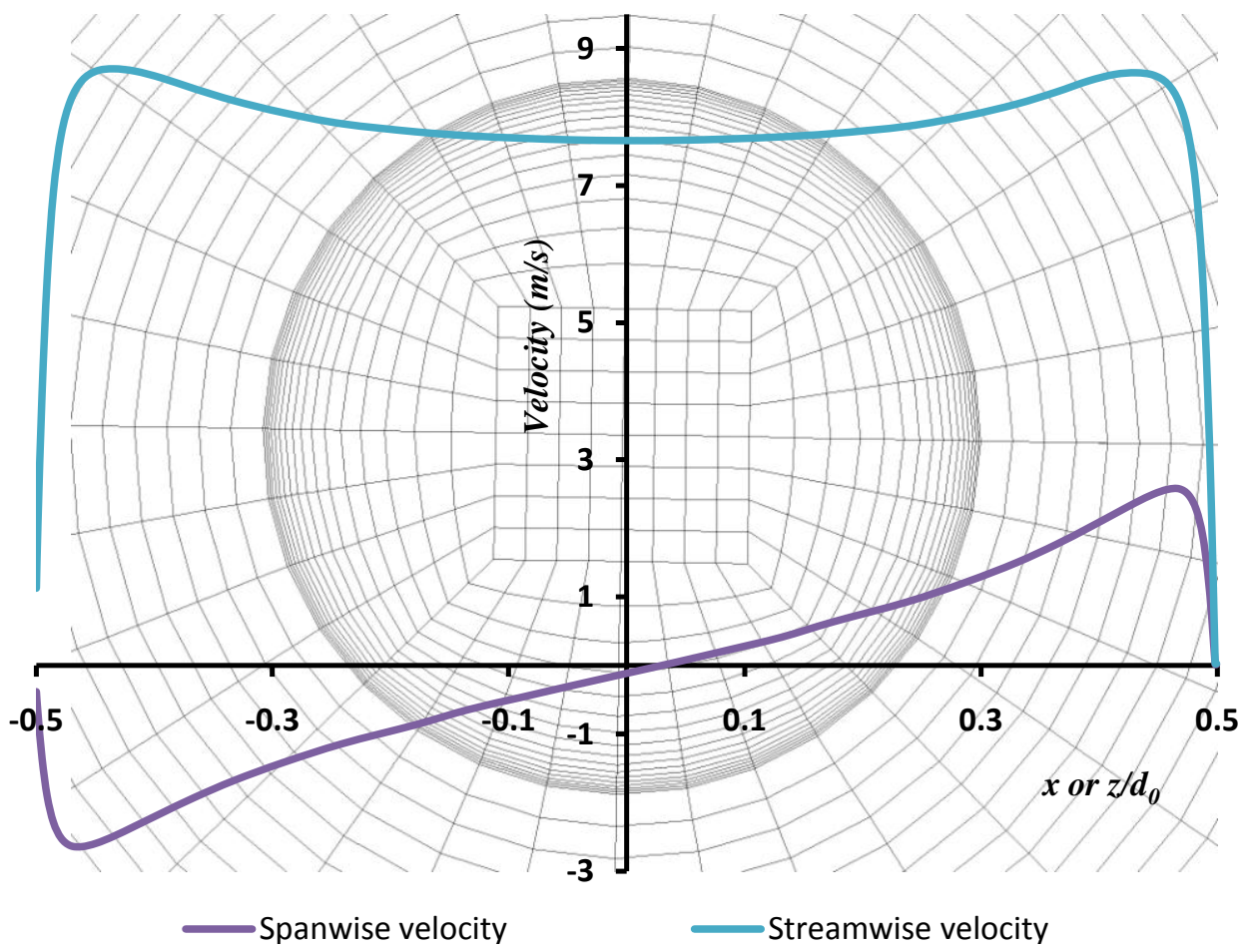

Figure 1. Inlet grid and its velocity profile

Another question concerns how to simulate laminar, transition and turbulent regions of free round jet with a single turbulence model. The following summarizes the various methods that can be applied to predict all regions of round jets. A. Direct Numerical Simulation (DNS) can obviously resolve all the flow scales, but is limited to computer resources. B. Large Eddy Simulation (LES) gives promising results for proximal region of round jet [1], while LES is sophisticated, costly and more time consuming for engineers than Reynolds Averaged Navier-Stokes (RANS) models. Olsson and Fuchs [1] briefly stated that "Most turbulence models are not capable of modeling the transition from laminar to turbulent flow directly". They [1] also presented LES results on proximal region of round jet. C. If the transition point is known from DNS or experiments, it is possible to generate mesh for the separated laminar region and resolve it by RANS models; this is called the indirect method. D. Another technique of employing RANS models for resolving transition from laminar to turbulence is called "low Reynolds" turbulence approach, even though they are tuned for high Reynolds number flows. Both the Low Reynolds number $k-\epsilon$ model (Launder and Sharma [34]) and the Low Reynolds Shear Stress Transport (SST) $k-\omega$ model (Menter [35]) are examples of low Reynolds RANS models. E. Two transition models $((k-k l-\omega)[36,37]$ and $\operatorname{TrSST} k-\omega$ [38]) have recently been presented that resolve extra equations in order to capture transition of the boundary layer from laminar to a turbulent regime.

When free round jets are issuing from different parallel-coaxial apertures in the same plane flow, they coalesce or merge with each other and move as a single jet at a certain distance downstream [39-41], which is called confluent jets. This coalescence probably causes more momentum survival of the issuing jets. Proximal regions of free round jets contain recirculation due to entrainment and transition to self-similar turbulence, which become more complicated for confluent round jets due to converging, merging and combining of fluid streamlines. These confluent jets exist in different applications such as ventilation systems, thrust and noise control of S/VTOL aircrafts, cooling of devices (e.g. film cooling of turbine blades or electronic devices), effluents in sewage water, combustion nozzles or fuel injection, fuel-air mixing and dilution holes in gas turbines, burners or chemical reactors and pollutant dispersion from chimney stacks. Based on the authors' knowledge, the whole field of round jet is only simulated in two dimensions with RANS turbulence models by Gouldin et al [28], Quinn and Militzer [2] and 
Faghani et al. [42]. The two-dimensional simulation with RANS models is not possible for confluent jets due to asymmetry in boundary conditions of round jets; it is now time to check the realism of three-dimensional prediction of the whole domain (especially initial and transitional regions) of low-Reynolds round jet with RANS turbulence models.

In the present paper, results of three-dimensional modeling of isothermal, free, turbulent round jet with two twoequation (Low Re $k-\epsilon$ and SST $k-\omega$ ), a transition three-equation $(k-k l-\omega)$ and a transition four-equation (SST) eddy-viscosity turbulence models with resolved inlet profiles are compared and validated with hot-wire anemometry. To the authors' knowledge, this prediction of free round jets is probably one of the first studies that cover the whole three-dimensional domain (initial, transitional and turbulent regions) by using RANS turbulence models.

\section{Computational set-up and numerical scheme}

All The computational domain is an incomplete conical frustum where the nozzle exit is placed at the center of the smallest face. The round jet is assumed unventilated (with front plate) due to short height of contraction. Reynolds numbers $\left(R e_{d_{0}}\right)$ of nozzle exit based on bulk velocities are 2125, 3461 and 4554.

The research finite-volume solver Fluent 12.1.4 was employed to numerically simulate the airflow of a threedimensional round jet. The governing and transport equations are solved with segregated memory-efficient algorithms. The double precision, steady state and pressure-based solver is used for the Low Re $k-\epsilon$ and SST $k-\omega$ models. Regarding discretization, the non-linear terms are calculated with the Second-order upwind scheme and the viscous terms with the Second-order central scheme. The under-relaxation factor for the pressure, momentum, turbulent kinetic energy and turbulent dissipation rate (specific dissipation rate) is set to $0.3,0.7,0.5$ and 0.5 respectively. The SIMPLE algorithm solves the pressure-velocity coupling. If the sum of absolute normalized residuals of transport equations in turbulent models for all of the cells in flow domain is below $10^{-6}$, the solutions were considered converged.

The computational staggered grid is generated with hexahedral cells so that the total number is below one million cells for the selected case based on grid independency. The mesh is refined enough near the solid wall, where the wall nearest $y^{+}$is kept below one to solve all boundary layers compatible with Low Reynolds correction. Special attention has also been paid to controlling the dimensionless streamwise, normal and span-wise spacing, i.e. $x^{*}, y^{*}$ and $z^{*}$, in order to avoid the generation of cells with high aspect ratio, which would degrade the numerical accuracy. The model decomposition and structured mesh is generated with Gambit 2.4.6.

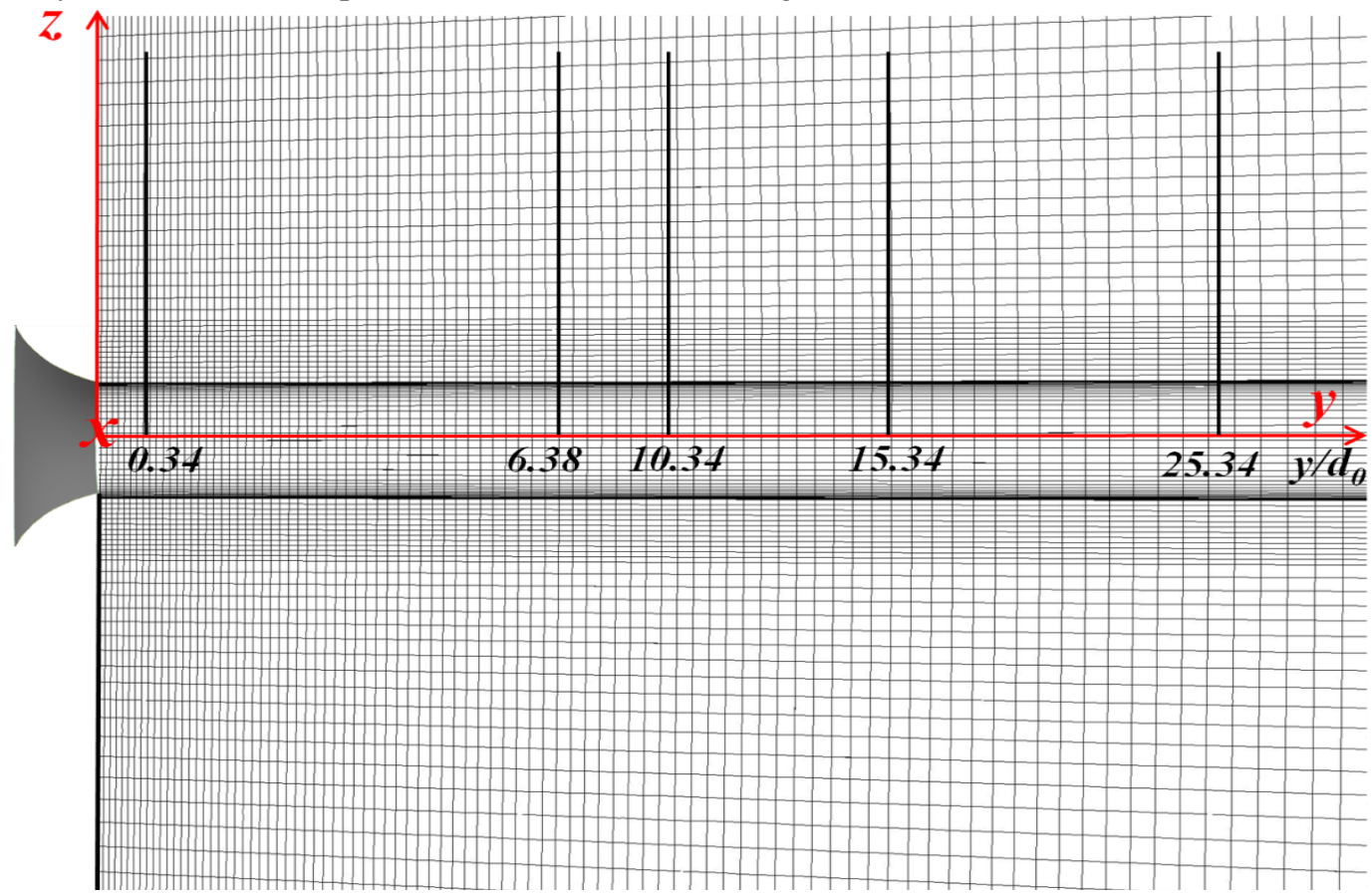

Figure 2. Sketch of round jet and coordinate system (not scaled) 
The isothermal (no buoyancy effect) airflow is governed by conservation laws of mass and momentum. The air is modeled as an ideal gas. Based on the above assumptions, the averaged continuity and Reynolds equations for steady-state, three-dimensional, incompressible and turbulent conditions are given by

$$
\begin{gathered}
\frac{\partial U_{i}}{\partial x_{j}}=0 \\
\frac{\partial\left(U_{j} U_{i}\right)}{\partial x_{j}}=-\frac{1}{\rho} \frac{\partial p}{\partial x_{i}}+\frac{\partial}{\partial x_{j}}\left(v \frac{\partial U_{i}}{\partial x_{j}}-\overline{u_{i}^{\prime} u_{j}^{\prime}}\right)
\end{gathered}
$$

where $\overline{u_{i}^{\prime} u_{j}^{\prime}}$ is the second moment of statistical correlation that is called the Reynolds stress and must be modeled in order to close the system of equations. A popular, common approach to modeling Reynolds stresses of equation (3), is applying the Boussinesq hypothesis [43], which relates them to mean velocity gradients as follows:

$$
-\overline{u_{i}^{\prime} u_{j}^{\prime}}=v_{t}\left(\frac{\partial u_{i}}{\partial x_{j}}+\frac{\partial u_{j}}{\partial x_{i}}\right)-\frac{2}{3}\left(k+v_{t} \frac{\partial u_{k}}{\partial x_{k}}\right) \delta_{i j}
$$

The Boussinesq hypothesis is employed in the Low Reynolds number $k-\epsilon$ model, the Low Reynolds Shear Stress Transport (SST) $k-\omega$ model, Transition $k-k l-\omega$ and Transition SST $k-\omega$. Only two additional transport equations for the turbulence kinetic energy, $k$, and turbulence dissipation rate, $\epsilon$ or specific dissipation rate, $\omega$ needs to be solved. Therefore $v_{t}$ is assumed as an isotropic scalar quantity and computed as a function of $k$ and $\epsilon$ (or $\omega$ ). This approach results in relatively low computational cost associated with modeling of turbulent viscosity $\left(v_{t}\right)$.

A general form for all the above-mentioned turbulence model equations can be written as follows:

$$
\frac{\partial \bar{\phi}}{\partial t}+\overline{u_{j}} \frac{\partial \bar{\phi}}{\partial x_{j}}-\frac{\partial}{\partial x_{j}}\left[\Gamma_{\phi, e f f} \frac{\partial \bar{\phi}}{\partial x_{j}}\right]=S_{\phi}
$$

where $\phi$ represents variables, $\Gamma_{\phi, e f f}$ is the effective diffusion coefficient, and $S_{\phi}$ represents the source term of an equation. The low Re $k-\epsilon$ model consists of the three modified damping functions, $f_{\mu}, C_{\epsilon 1}^{*}$ and $C_{\epsilon 2}^{*}$, and two additional terms ( $D$ and $E$ ) compared to standard $k-\epsilon$. These major modifications to Low Re $k-\epsilon$ model improve the performance in places with low Re number. In the Low Re SST $k-\omega, Y$ represents the dissipation term in the $k$ and $\omega$ equations while $F_{1}$ and $F_{2}$ are blending functions that control the switch between $k-\epsilon$ model and standard $k-\omega$ model. $D_{\omega}$ is generated from the $k-\epsilon$ model and it vanishes in the $k-\omega$ model when the blending function $F_{1}$ equals to one.

Two-equation Reynolds-Averaged Navier-Stokes (RANS) turbulence models with Boussinesq approximation simulate the flow as fully turbulent. Additional equations, which are not necessarily required with Boussinesq approximation to solve closure problems, may result in more accurate prediction of laminar, transition from laminar to turbulent and turbulent regimes (especially the onset of transition). Two different transition RANS turbulence models, $k-k l-\omega$ and TrSST $k-\omega$, are included in the round jet study. These turbulence models were mostly tuned for prediction of transition in wall boundary layers. In the transition $k-k l-\omega$ model, transport equations of turbulent kinetic energy, $k_{T}$, and laminar kinetic energy, $k_{L}$, are separately solved. These two transport equations together with the transport equation of the specific dissipation rate, $\omega$, make a three-equation closed system and determine the turbulent eddy viscosity, $v_{t}=\frac{k_{L}+k_{T}}{\omega} . k_{L}$ and $k_{T}$ represent the pre-transitional (laminar) velocity fluctuations and magnitude of fluctuations of fully turbulent flow, respectively. The production of $k_{L}$ is assumed from the interactions between mean shear and pre-transitional velocity fluctuations. Then an energy transfer from $k_{L}$ to $k_{T}$ expresses the transition process. The onset of transition is assumed to arise at sufficiently short time-scale of turbulence- production dynamics compared to time-scale of molecular diffusion. The Low Re SST $k-\omega$ uses damping functions of the underlying turbulence model in order to predict transition. A local correlation-based transition modeling has been proposed by coupling two new equations to the original Low Re SST $k-\omega$, thus making a four-equation model. One transport equation for intermittency, $\gamma$, determines the onset of transition, while one transport equation for the transition momentum thickness Reynolds number, $R e_{\theta t}$, as a scalar quantity is included to prevent additional nonlocal operations. $R e_{\theta t}$ depends on the local and free stream turbulent intensities, which is attributed to diffusion of experimentally-corrected free stream value of $R e_{\theta t}$ into the boundary layer. 


\section{Experimental set-up}

The size of the well-insulated test room is $4.2 \times 3.6 \times 2.5 \mathrm{~m}^{3}$, where temperature can be controlled within $0.1{ }^{\circ} \mathrm{C}$. The test rig is located inside of an operating room that is separated by insulation from the main laboratory ambient. Wooden walls of test room include a water pipe system for temperature control. The airflow feed issues from a centrifugal fan with straight radial blade. A frequency regulator controlled the blower power in order to get the desired airflow rate. Supply air duct is connected to the fan and an orifice meter. Then a flexible-rubber duct is connected to the straight $2 \mathrm{~m}$ long aluminum pipe to absorb mechanical vibrations. At the end of the long pipe, the model is placed. No filter such as honeycomb was used for this measurement. The air exits the test room without forcing through a rectangular exhaust on one of the sidewalls.

Nozzle (see Figure 1 and Figure 2) with exit diameter $\left(d_{0}\right) 0.0058 \mathrm{~m}$ is located on a cylindrical air supply, which is placed at the center of the longest wall of the test room. Nozzle exit was designed so that its inlet profile differs from top-hat-like velocity profile (see Figure 1). Nozzle contraction with small aspect ratio causes an increase in centerline velocity and a decrease in the velocity on the jet borders in the downstream region of the nozzle. Entrainment of nominally static fluid surrounding the nozzle exit can also amplify the contraction effect with an increase in radial momentum.

Longitudinal velocity component in cross-section of the nozzle was measured from a round nozzle at Reynolds number 3461 by using a single-wire sensor. Every set of measurements includes a series of data along the cross section of nozzles at different distances ranging from 0.06 to $50 d_{0}$ downstream from the exit section with separation ranging $0.017 d_{0}$. Each measurement covered the complete profile up to $10 \%$ of the maximum centerline velocity. Every data point was logged at $5 \mathrm{kHz}$ for 180 seconds.

Measured turbulence levels during this work is less than $50 \%$, which Bruun [47] showed that the problem of rectification is very limited below this limit considering homogeneous and isotropic turbulence. Relative expanded uncertainty of velocity measurements by the single-sensor hot-wire probe in air is $4.23 \%$. Sources of uncertainty and their relative standard uncertainty can be summarized as follows: calibration (2\%), linearization (curve fitting) $(0.5 \%)$, Analogue/Digital board resolution $(0.13 \%)$, probe positioning $(\approx 0)$, air and sensor temperature variations $(1 \%)$, ambient pressure variations $(0.6 \%)$ and humidity $(\approx 0)$.

Centerline of the jet flow has low turbulence intensity up to almost two times nozzle diameter, and then a steep initial rise in turbulence intensity (Figure 3) is observed which is brought about by turbulence production from shear layers of mean flow and transport by diffusion to the jet centerline. After almost seven times nozzle diameter, centerline flow changes its behavior and its turbulence intensity follows a gradual rise which means the flow starts to become self-similar.

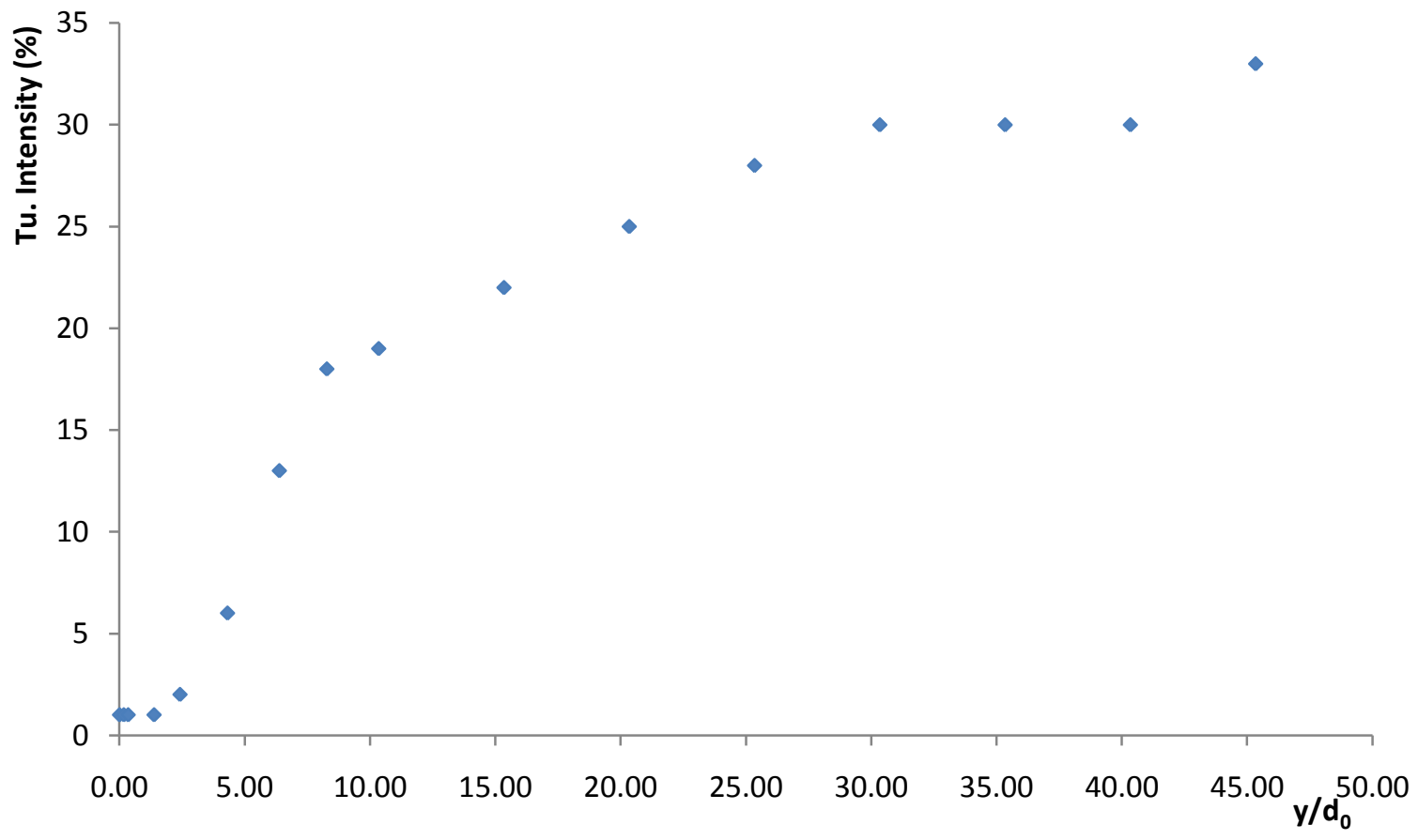

Figure 3. Measured turbulence intensity of streamwise velocity along centerline 
For all cross sections, skewness and flatness of streamwise velocity around jet axis are observed very close to Gaussian values (zero and three, respectively). This is also consistent with centerline measurement. The skewness factor and flatness factor indicate that the turbulence velocity distribution close to centerline is almost the same as Gaussian probability density distribution and their deviations from Gaussian level for both factors in radial direction (more pronounced in the downstream direction) is attributed to the intermittency of the jet flow.

\section{Results}

Analytical analysis [23] of momentum conservation in fully developed region of round jet proves the empirical law that product of jet's half width $\left(r_{1 / 2}\right)$ and centerline velocity does not function of distance from nozzle $(y)$, i.e. $r_{1 / 2} \propto y$ and $U_{\text {centerline }} \propto y^{-1}$. Prediction of round jet with three Reynolds numbers, based on the jet exit diameter and velocity, $(2125,3461$ and 4554$)$ is consistent with the mentioned axial variation of centerline velocity. From now on because the overall behavior of mean flow characteristics overlap for all three Reynolds numbers, the results of three-dimensional numerical simulation with correctly predicted inlet boundary conditions of Reynolds number 3461 is demonstrated here.

Fluid streamlines of free round jet are unable to carefully follow sharp curves or direction change of short contoured nozzle. This phenomenon is called vena contracta and shows up in contractions and causes a least convergence diameter of streamlines just downstream of nozzle exit due to inward direction (toward the jet centerline) of mean radial velocities. The amplified effect can occur in this study due to flush (abrupt transition of the flow) from short contracting nozzle exit within the test room. This configuration brings entrainment of nominally static fluid surrounding the nozzle exit into initial and transitional regions of jet development with radial momentum and contribution to concentration of streamwise momentum in the centerline of the jet. Verification between measurements and numerical prediction of mean velocity profile in $x$ direction for the nozzle is illustrated in Figure 4. Predicted mean velocity profile in $x$ direction complies with measurements at $0.34 d_{0}$ from nozzle's exit plane. Velocity profile at this cross section shows a top-hat profile and in the outer part of centerline contains overshoot that is attributed to small convergence in nozzles, which cause an increase in centerline velocities (vena contracta phenomena).

Figure 1 shows that curvature of streamlines of the mean streamwise velocity profiles at the exit plane is initiated within contraction and does not end at this plane. Centrifugal acceleration of fluid particles occurs due to the streamline curvature. The resulting centrifugal forces must then be balanced by a transverse pressure gradient. Such a streamline curvature is absent in flows from parallel exits.

Measured turbulence intensity profile of nozzle along $x$ direction at $0.34 d_{0}$ from the nozzle's exit plane is presented in Figure 4 . This quantity has a very small value $(\approx 1 \%)$ throughout core part (constant-velocity region); on the contrary, turbulence intensity slightly rises to a maximum lying around $10 \%$. This limited turbulence intensity confirms existence of laminar boundary layer at the exit of nozzles.

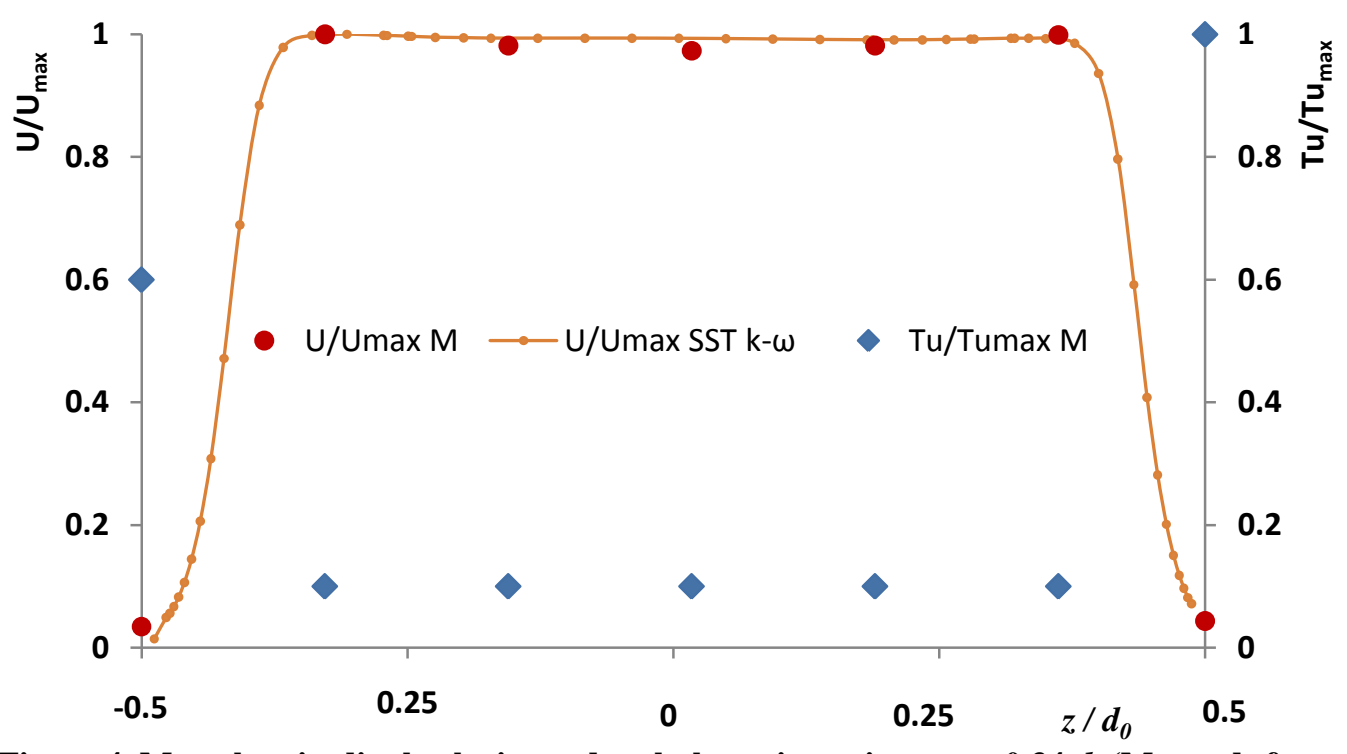

Figure 4. Mean longitudinal velocity and turbulence intensity at $y=0.34 d_{0}$ (M stands for measurement) 
The vena contracta effect can evidently be seen in the close-up view of mean streamwise velocity along the centerline of jet (Figure 5), i.e. the initial region is influenced by nonparallel nozzle exit. The jet shows a significant acceleration along its centerline. This fluid particle acceleration on the jet centerline in the initial region is directly attributable to the mean radial velocity.

There is excellent agreement between experimental and numerical results by Low Reynolds turbulence models but the two transition models could not completely predict decay of centerline velocity in all regions (except distances from zero to $4.5 d_{0}$ ).

The numerical results of centerline velocity decay in turbulent region are consistent with the function mentioned in the introduction (1). The velocity profile (see Figure 6) in the developed region of the axisymmetric round jet can be modeled by a Gaussian distribution, $U / U_{\max }=\exp \left[-A^{2}\left(x /\left(y-y_{p}\right)^{2}\right]\right.$, where $A=(0.5 \ln 2)^{0.5} / \tan \beta$ and $\tan \beta=x_{0.5} /\left(y-y_{p}\right)$ and $y_{p}$ is the distance from nozzle exit to the virtual origin of the jet. In Table 2 decay of high and low velocity axisymmetric round jets are compared. Malmström et al. [44] re-evaluated the results of Nottage [45] and then compared them with their own experimental data, concluding that A-value decreases (or halfwidth spreading angle $\beta$ increases) at low nozzle Reynolds number. Their conclusion is consistent with the present study.

Table 2. Comparison of high and low velocity round jets decay

\begin{tabular}{lccllll}
\hline & $U_{0}(\mathrm{~m} / \mathrm{s})$ & $d_{0}(\mathrm{~m})$ & $\mathrm{Re}$ & $\tan \beta$ & \multicolumn{1}{c}{$A$} & $y_{p} / d_{0}$ \\
\hline \hline Nottage [44, 45] & 5.1 & 0.1524 & 51000 & 0.109 & 5.42 & 2.6 \\
Wygnanski \& Fiedler [15] & 51 & 0.0254 & 100000 & 0.086 & 5.7 & 3 \\
Rodi [16] & 101 & 0.012 & 87000 & 0.086 & 5.9 & \\
Panchapakesan \& Lumley [17] & 27 & 0.61 & 11000 & 0.096 & 6.06 & -2.5 \\
Hussein et al. LDA [18] & 56.2 & 0.0254 & 95500 & 0.094 & 5.8 & 4 \\
Hussein et al. HWA [18] & 56.2 & 0.0254 & 95500 & 0.102 & 5.9 & 2.7 \\
Malmström et al. [44] & 8.7 & 0.0401 & 19000 & 0.095 & 6.22 & -1.6 \\
Present study & 8.3 & 0.0058 & 3400 & 0.109 & 5.37 & -1.75 \\
\hline \hline
\end{tabular}

Longitudinal (streamwise) mean velocity variation with axial distance $(y)$ along the centerline of round jet is depicted in Figure 5. The mean velocity is normalized with maximum velocity along the centerline.

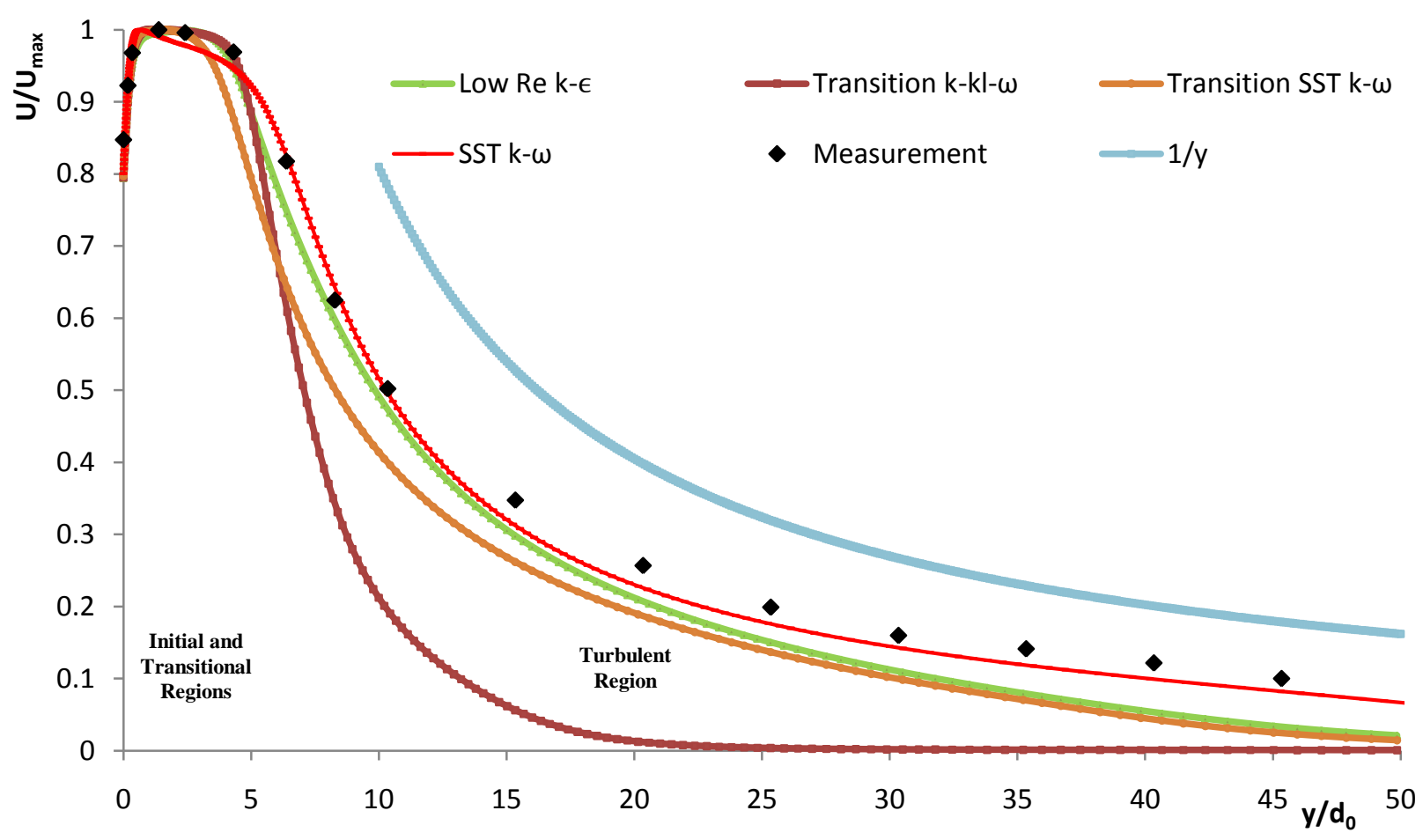

Figure 5. Centerline mean longitudinal velocity 
Predicted velocities in initial, transitional and turbulent regions from both low-Re turbulence models are more compatible with measurement results. The SST $k-\omega$ model is more consistent with hot-wire anemometry data, especially in the self-similar region.

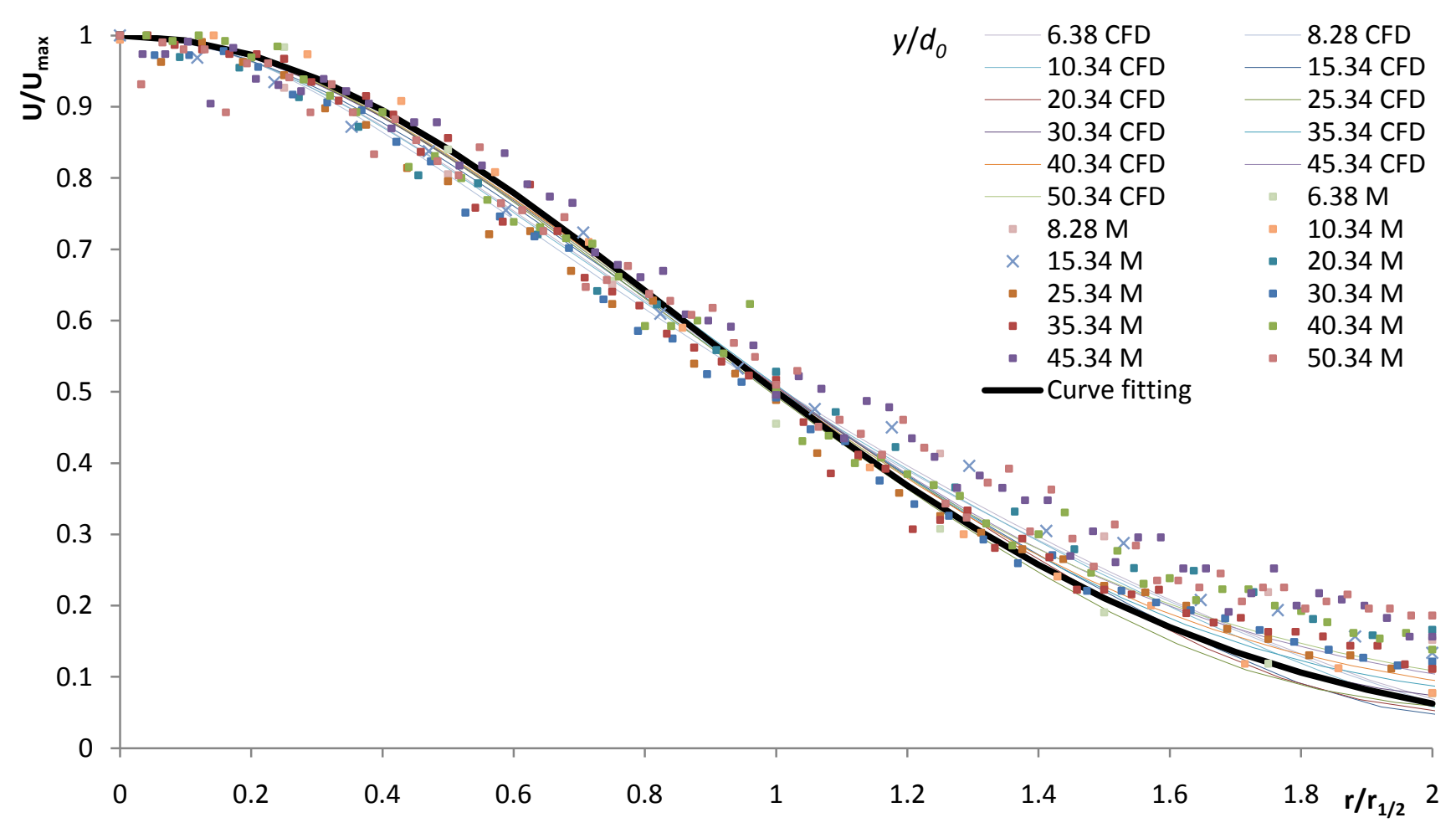

Figure 6. Self-similarity of round jet at different cross section profiles (M stands for measurement)

Figure 6 illustrates the self-similarity of velocity $\left(U / U_{\max }\right)$ versus $r / r_{1 / 2}$ for $y / d_{0} \geq 6.38$. It is deducible from Figure 6 that the round jet keeps its shape while the mean longitudinal velocity decays and the jet's half width, $\left(r_{1 / 2}\right)$ increases with an increase in axial distance $(y)$. For the self-similar region of the round jet flow, which is $U \propto y^{-1}$, the non-dimensional velocity profile is often approximated by an equation: $U / U_{\max }=e^{\left[-C_{0}\left(r / r_{0.5}\right)^{2}\right]}$ represents a Gaussian distribution. According to Figure $6, C_{0}$ equals $\ln 2$ and evidently depends on nozzle geometry. Regarding reversal flow effects, the measurements plots are cut at $U / U_{\max } \approx 0.1$, in order to avoid the effects of induced entrainment transverse velocity and high fluctuation rates.

Due to difficulty of jet's spreading boundary determination, velocity half width, $r_{1 / 2}$, is a convenient scale for the jet's width, i.e. the radial distance from jet centerline to the place where $\mathrm{U}=0.5 \mathrm{U}_{\max }$. Figure 7 shows the values of $r_{1 / 2}$ for the $0 \leq y / d_{0} \leq 50$. All $r_{1 / 2} / d_{0}$ within $0 \leq y / d_{0} \leq 6.38$ come near to 0.5 , which certainly reflects the similarity between nozzle exit profile and those within initial and transitional regions. The precise values of $r_{1 / 2} / d_{0}$ at the nozzle can be determined from the data of simulated nozzle inlet boundary conditions (Figure 1), which is between 0.49 and 0.5 . A least square estimation can be written for the linear growth of axisymmetric jet in selfsimilar region: $r_{1 / 2} / d_{0}=0.111\left(y / d_{0}-0.96\right)$ for $y / d_{0} \geq 6.38$. The significant point to note is that the first moment self-similarity of the studied low-Reynolds-number jet begins at around $6 d_{0}$ (which for $\operatorname{Re}=10^{4}$ is reported $30 d_{0}$ [14] or for $\operatorname{Re}=10^{5}$ is $20 d_{0}$ [15] or for $\operatorname{Re}=1.3 \times 10^{4}$ is $4 d_{0}$ [6]). Linear constant spreading rate is about 0.111 (reported spreading rate of high Reynolds round jets by Hussein [18] (Re= 95500: LDA: 0.094 and HWA: 0.102) and Panchapakesan [17] (Re=11000: HWA: 0.096)). 


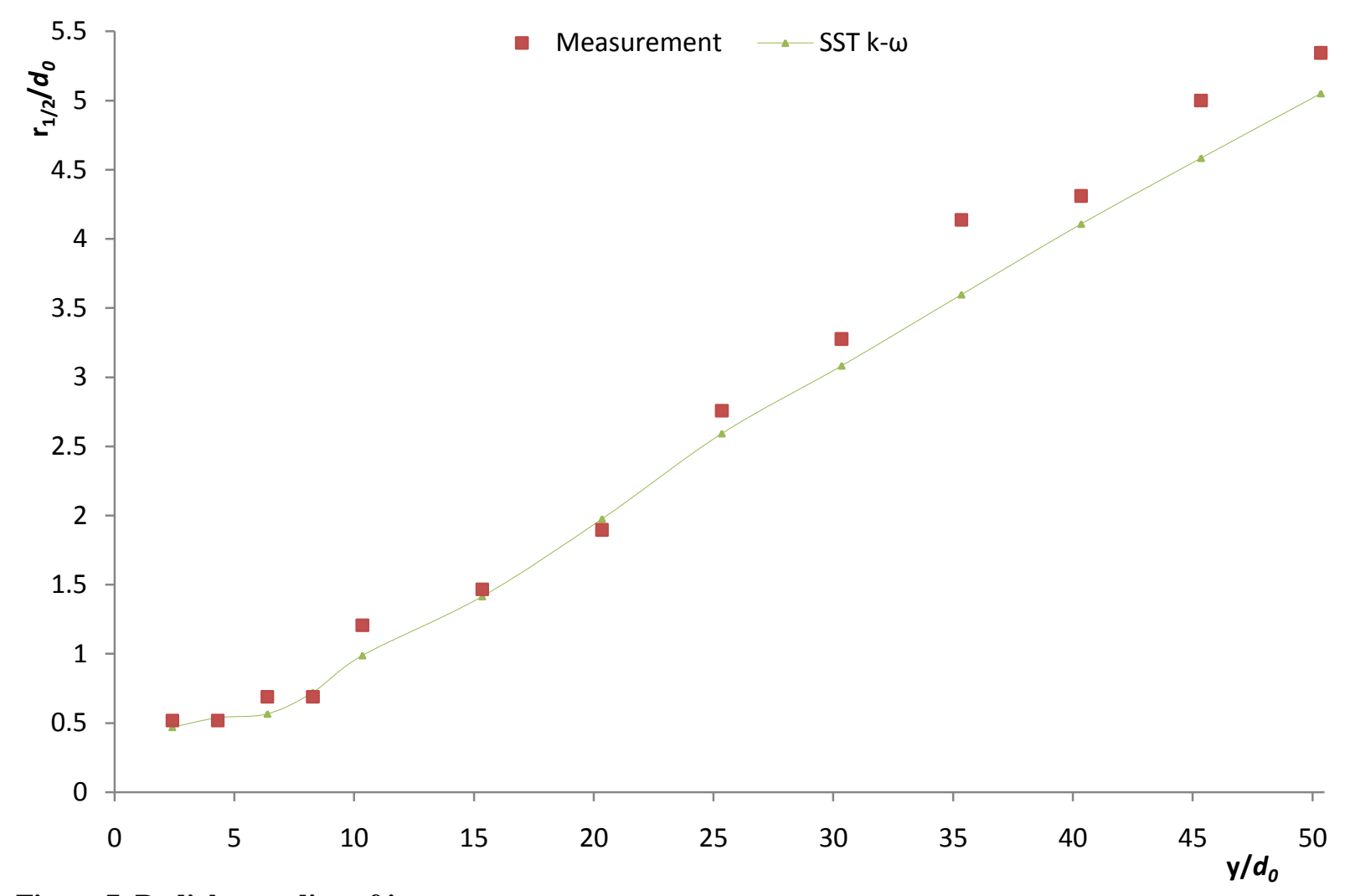

Figure 7. Radial spreading of jet

\section{Conclusion}

This study evaluated the performance of some RANS turbulence models in three-dimensional simulation of the whole field (laminar, transition and turbulent regions) of round jet. It was also shown that providing the inlet boundary condition of the round jet plays an important role in prediction of proximal regions.

Among employed RANS models, Low Reynolds turbulence models (the Low Re number $k-\epsilon$ and the Low Re SST $k-\omega)$ show the best overall performance in order to predict the whole field compared to transition models in terms of accuracy, computing efficiency and robustness. Both low Re models are recommended for low Re free round jet simulations. The Low Re SST $k-\omega$ model gave better accuracy in simulation without increasing computing time compared to the Low Re number $k-\epsilon$ model.

The transition three-equation $(k-k l-\omega)$ and the transition four-equation (SST) eddy-viscosity turbulence models did not perform well enough in fully turbulent region of the round jet. These two transition models performed reasonably well in the proximal region (laminar and transition regions).

\section{References}

${ }^{1}$ Olsson, M., and Fuchs, L. "Large eddy simulation of the proximal region of a spatially developing circular jet," Physics of Fluids Vol. 8, No. 8, 1996, pp. 2125-2137.

${ }^{2}$ Quinn, W. R., and Militzer, J. "Effects of nonparallel exit flow on round turbulent free jets," International Journal of Heat and Fluid Flow Vol. 10, No. 2, 1989, pp. 139-145.

doi: 10.1016/0142-727x(89)90008-8

${ }^{3}$ Sami, S., Carmody, T., and Rouse, H. "Jet diffusion in the region of flow establishment," Journal of Fluid Mechanics Vol. 27, No. 02, 1967, pp. 231-252. doi: $10.1017 / \mathrm{S} 0022112067000291$

${ }^{4}$ Hill, B. J. "Measurement of local entrainment rate in the initial region of axisymmetric turbulent air jets," Journal of Fluid Mechanics Vol. 51, No. 04, 1972, pp. 773-779.

doi: 10.1017/S0022112072001351 
${ }^{5}$ Boguslawski, L., and Popiel, C. O. "Flow structure of the free round turbulent jet in the initial region," Journal of Fluid Mechanics Vol. 90, No. 03, 1979, pp. 531-539.

doi: 10.1017/S0022112079002378

${ }^{6}$ Obot, N. T., Graska, M. L., and Trabold, T. A. "The near field behavior of round jets at moderate reynolds numbers," The

Canadian Journal of Chemical Engineering Vol. 62, No. 5, 1984, pp. 587-593.

doi: 10.1002/cjce.5450620503

${ }^{7}$ Davies, P. O. A. L., Fisher, M. J., and Barratt, M. J. "The characteristics of the turbulence in the mixing region of a round

jet," Journal of Fluid Mechanics Vol. 15, No. 03, 1963, pp. 337-367. doi: 10.1017/S0022112063000306

${ }^{8}$ Bradshaw , P., Ferriss, D. H., and Johnson, R. F. "Turbulence in the noise-producing region of a circular jet," Journal of

Fluid Mechanics Vol. 19, No. 04, 1964, pp. 591-624. doi: 10.1017/S0022112064000945

${ }^{9}$ Ko, N. W. M., and Davies, P. O. A. L. "The near field within the potential cone of subsonic cold jets," Journal of Fluid

Mechanics Vol. 50, No. 01, 1971, pp. 49-78. doi: $10.1017 / \mathrm{S} 0022112071002453$

${ }^{10}$ Lau, J. C., and Fisher, M. J. "The vortex-street structure of 'turbulent' jets. Part 1," Journal of Fluid Mechanics Vol. 67, No. $02,1975$.

doi: $10.1017 / \mathrm{S} 0022112075000328$

${ }^{11}$ Crow, S. C., and Champagne, F. H. "Orderly structure in jet turbulence," Journal of Fluid Mechanics Vol. 48, No. 3, 1971, pp. 547-591. doi: $10.1017 / \mathrm{S} 0022112071001745$

${ }^{12}$ Hussain, A. K. M. F. "Coherent structures---reality and myth," Physics of Fluids Vol. 26, No. 10, 1983, pp. $2816-2850$. doi: $10.1063 / 1.864048$

${ }^{13}$ Dimotakis, P. E., Miake-Lye, R. C., and Papantoniou, D. A. "Structure and dynamics of round turbulent jets," Physics of Fluids Vol. 26, No. 11, 1983, pp. 3185-3192.

${ }^{14}$ Pope, S. B. Turbulent Flows: Cambridge University Press, 2000.

${ }^{15}$ Wygnanski, I., and Fiedler, H. "Some measurements in the self-preserving jet," Journal of Fluid Mechanics Vol. 38, No. 3, 1969, pp. 577-612.

doi: $10.1017 / \mathrm{S} 0022112069000358$

${ }^{16}$ Rodi, W. "A new method of analysing hot-wire signals in highly turbulent flow, and its evaluation in a round jet," DISA Information Vol. 17, 1975, pp. 9-18.

${ }^{17}$ Panchapakesan, N. R., and Lumley, J. L. "Turbulence measurements in axisymmetric jets of air and helium. Part 1. Air jet," Journal of Fluid Mechanics Vol. 246, No. -1, 1993, pp. 197-223.

doi: 10.1017/S0022112093000096

${ }^{18}$ Hussein, J. H., Capp, S. P., and George, W. K. "Velocity measurements in a high-Reynolds-number, momentumconserving, axisymmetric, turbulent jet," Journal of Fluid Mechanics Vol. 258, 1994, pp. 31-75.

doi: 10.1017/S002211209400323X

${ }^{19}$ Rajaratnam, N. Turbulent Jets. Amsterdam and New York: Elsevier Publishing Co., 1976.

${ }^{20}$ Schlichting, H., and Gersten, K. Boundary-Layer Theory Springer, 2000.

${ }^{21}$ Spalding, D. B. "Concentration fluctuations in a round turbulent free jet," Chemical Engineering Science Vol. 26, No. 1,

1971, pp. 95-107.

doi: 10.1016/0009-2509(71)86083-9

22. Launder, B. E., Morse, A., Rodi, W., and Spalding, D. B. "Prediction of free shear flows: A comparison of the performance of six turbulnec models." Vol. NASA SP - 311, 1972.

${ }^{23}$ Pope, S. B. "An explanation of the turbulent round-jet/plane-jet anomaly," AIAA Journal Vol. 16, 1978, pp. $279-281$. doi: $10.2514 / 3.7521$

${ }^{24}$ Givi, P., and Ramos, J. I. "On the calculation of heat and momentum transport in a round jet " International Communications in Heat and Mass Transfer Vol. 11, No. 02, 1984, pp. 173-182. doi: 10.1016/0735-1933(84)90020-4

${ }^{25}$ Cho, J. R., and Chung, M. K. "A k-E- $\gamma$ equation turbulence model," Journal of Fluid Mechanics Vol. 237, 1992, pp. 301322.

doi: doi:10.1017/S0022112092003422

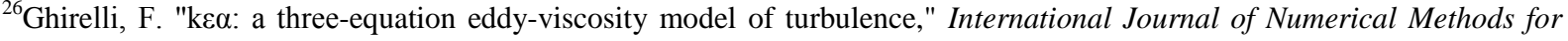
Heat \& Fluid Flow Vol. 17, No. 2, 2007, pp. 140-164.

doi: 10.1108/09615530710723939

${ }^{27}$ Zaman, K. B. M. Q., and Hussain, A. K. M. F. "Natural large-scale structures in the axisymmetric mixing layer," Journal of Fluid Mechanics Vol. 138, 1984, pp. 325-351.

doi: 10.1017/S0022112084000148

${ }^{28}$ Gouldin, F. C., Schefer, R. W., Johnson, S. C., and Kollmann, W. "Nonreacting turbulent mixing flows," Progress in Energy and Combustion Science Vol. 12, No. 4, 1986, pp. 257-303.

11

American Institute of Aeronautics and Astronautics 
doi: 10.1016/0360-1285(86)90004-3

${ }^{29}$ George, W. K. The self-preservation of turbulent flows and its relation to initial conditions and coherent structures. New York: Hemisphere, 1989.

${ }^{30}$ George, W. K., and Davidson, L. "Role of initial conditions in establishing flow behavior," AIAA Journal Vol. 42, No. 3, 2004, pp. 438-446.

doi: $10.2514 / 1.3459$

${ }^{31}$ Boersma, B. J., Brethouwer, G., and Nieuwstadt, F. T. M. "A numerical investigation on the effect of the inflow conditions on the self-similar region of a round jet," Physics of Fluids Vol. 10, No. 4, 1998, pp. 899-909.

doi: $10.1063 / 1.869626$

${ }^{32}$ Antonia, R. A., and Zhao, Q. "Effect of initial conditions on a circular jet," Experiments in Fluids Vol. 31, No. 3, 2001, pp. 319-323.

doi: $10.1007 / \mathrm{s} 003480100289$

${ }^{33}$ Ghahremanian, S., and Moshfegh, B. "Experimental and numerical verification of confluent jets Part I: Inlet boundary condition," (unpublished).

${ }^{34}$ Launder, B. E., and Sharma, B. I. "Application of the energy-dissipation model of turbulence to the calculation of flow near a spinning disc," Letters in Heat and Mass Transfer Vol. 1, No. 2, 1974, pp. 131-137.

${ }^{35}$ Menter, F. R. "Two-equation eddy-viscosity turbulence models for engineering applications," AIAA Journal Vol. 32, No. 8, 1994, pp. 1598-1605.

36. Walters, D. K., and Cokljat, D. "A Three-Equation Eddy-Viscosity Model for Reynolds-Averaged Navier--Stokes Simulations of Transitional Flow," Journal of Fluids Engineering Vol. 130, No. 12, 2008, pp. 121401-14.

${ }^{37}$ Walters, D. K., and Leylek, J. H. "A New Model for Boundary Layer Transition Using a Single-Point RANS Approach," Journal of Turbomachinery Vol. 126, No. 1, 2004, pp. 193-202.

doi: $10.1115 / 1.1622709$

${ }^{38}$ Menter, F. R., Langtry, R. B., Likki, S. R., Suzen, Y. B., Huang, P. G., and Volker, S. "A Correlation-Based Transition Model Using Local Variables---Part I: Model Formulation," Journal of Turbomachinery Vol. 128, No. 3, 2006, pp. 413-422.

${ }^{39}$ Tanaka, E. "The Interference of Two-Dimensional Parallel Jets : 1st Report, Experiments on Dual Jet," Bulletin of JSME Vol. 13, No. 56, 1970, pp. 272-280

${ }^{40}$ Tanaka, E. "The Interference of Two-Dimensional Parallel Jets : 2nd Report, Experiments on the Combined Flow of Dual Jet," Bulletin of JSME Vol. 17, No. 109, 1974, pp. 920-927

${ }^{41}$ Tanaka, E., and Nakata, S. "The Interference of Two-Dimensional Parallel Jets : 3rd Report, The Flow Region Near the Nozzle in Triple Jets," Transactions of the Japan Society of Mechanical Engineers Vol. 41, No. 342, 1975, pp. 537-545

${ }^{42}$ Faghani, E., Saemi, S., Maddahian, R., and Farhanieh, B. "On the effect of inflow conditions in simulation of a turbulent round jet," Archive of Applied Mechanics, 2010, pp. 1-15.

doi: 10.1007/s00419-010-0494-8

${ }^{43}$ Hinze, J. O. Turbulence: McGraw-Hill Companies, 1975.

${ }^{44}$ Malmström, T. G., Kirkpatrick, A. T., Christensen, B., and Knappmiller, K. D. "Centreline velocity decay measurements in low-velocity axisymmetric jets," Journal of Fluid Mechanics Vol. 346, 1997, pp. 363-377.

doi: doi:null

${ }^{45}$ Nottage, H. B. "Report on ventilation jets in room air distribution." Case Inst. of Technology, Cleveland, Ohio, 1951.

${ }^{46}$ Capp, S. P., Hussein, J. H., and George, W. K. "Velocity measurement in a high Reynolds number, momentum conserving, axisymmetric, turbulent jet." University of Buffalo, Buffalo, 1990.

${ }^{47}$ Bruun, H. H. "Hot-Wire Anemometry - Principles and Signal Analysis ". Oxford University Press, 1995. 